\title{
Disease Diagnosis System Using IoT Empowered with Fuzzy Inference System
}

\author{
Talha Mahboob Alam ${ }^{1, *}$, Kamran Shaukat ${ }^{2,6}$, Adel Khelifi ${ }^{3}$, Wasim Ahmad Khan ${ }^{4}$, \\ Hafiz Muhammad Ehtisham Raza ${ }^{5}$, Muhammad Idrees ${ }^{6}$, Suhuai Luo ${ }^{2}$ and Ibrahim A. Hameed
}

\author{
${ }^{1}$ Department of Computer Science and Information Technology, Virtual University of Pakistan, Lahore, Pakistan \\ ${ }^{2}$ School of Information and Physical Sciences, The University of Newcastle, Australia \\ ${ }^{3}$ Department of Computer Science and Information Technology, Abu Dhabi University, Abu Dhabi, United Arab Emirates \\ ${ }^{4}$ School of Computer Science, National College of Business Administration \& Economics, Lahore, Pakistan \\ ${ }^{5}$ Sir Syed College of Computer Science, University of Engineering and Technology, Lahore, Pakistan \\ ${ }^{6}$ Department of Data Science, University of the Punjab, Lahore, Pakistan \\ ${ }^{7}$ Department of ICT and Natural Sciences, Norwegian University of Science and Technology, Trondheim, Norway \\ *Corresponding Author: Talha Mahboob Alam. Email: talhamahboob95@gmail.com \\ Received: 20 May 2021; Accepted: 05 August 2021
}

\begin{abstract}
Disease diagnosis is a challenging task due to a large number of associated factors. Uncertainty in the diagnosis process arises from inaccuracy in patient attributes, missing data, and limitation in the medical expert's ability to define cause and effect relationships when there are multiple interrelated variables. This paper aims to demonstrate an integrated view of deploying smart disease diagnosis using the Internet of Things (IoT) empowered by the fuzzy inference system (FIS) to diagnose various diseases. The Fuzzy System is one of the best systems to diagnose medical conditions because every disease diagnosis involves many uncertainties, and fuzzy logic is the best way to handle uncertainties. Our proposed system differentiates new cases provided symptoms of the disease. Generally, it becomes a time-sensitive task to discriminate symptomatic diseases. The proposed system can track symptoms firmly to diagnose diseases through IoT and FIS smartly and efficiently. Different coefficients have been employed to predict and compute the identified disease's severity for each sign of disease. This study aims to differentiate and diagnose COVID-19, Typhoid, Malaria, and Pneumonia. This study used the FIS method to figure out the disease over the use of given data related to correlating with input symptoms. MATLAB tool is utilised for the implementation of FIS. Fuzzy procedure on the aforementioned given data presents that affectionate disease can derive from the symptoms. The results of our proposed method proved that FIS could be utilised for the diagnosis of other diseases. This study may assist doctors, patients, medical practitioners, and other healthcare professionals in early diagnosis and better treat diseases.
\end{abstract}

Keywords: Disease diagnosis system; COVID-19; healthcare; biomedical; rules extraction 


\section{Introduction}

A cluster of pneumonia cases arose in Wuhan in China at the end of 2019 [1]. Investigations showed that it was caused by a previously unknown virus now called COVID-19. Hundreds of laboratories reported China's cases in January 2020, and the number of infections has risen daily. The disease spread swiftly from Wuhan to other cities. Most of the records are from Hubei province and different regions and municipalities of China that proclaimed similar cases [2,3]. COVID-19 is the root of one-third of the population that acquired upper respiratory infections in adults during an outbreak. They are also the source of severe respiratory infections in both adults and children. These types of cases are also reported in other countries, including Asiatic and European countries. COVID-19 is a broad community of viruses containing a nucleus of genetic material surrounded by protein spikes envelope that gives crown appearance [3]. Airborne illness might spread from common cold to pneumonia, and symptoms tend to be mild in most individuals.

Severe Acute Respiratory Syndrome, first described by China in 2003, and Middle East Respiratory Syndrome, first by Saudi Arabia in 2012, are coronavirus forms that cause serious illness [4]. The COVID-19 has a near resemblance to the bat coronaviruses and is known as bats are the primary source. However, the origins of COVID-19 remain under investigation. Recent evidence indicates that the transmission has spread to humans from illicit wildlife sold in the Hunan Seafood Wholesale Market. The first time COVID-19 was reported in China in 2019 and initially occurred in a group of pneumonia associated people in the city of Wuhan with seafood and animal markets [5]. Since then, this disease has spread to others, including family members and healthcare staff. Currently, there are many cases, and the disease is spreading throughout China and other countries. North America has the highest percentage of confirmation rate of COVID-19, and Europe has the highest percentage of death cases due to COVID-19. The current situation is complicated these days worldwide because Cough and Flu are considered coronavirus [6].

Artificial Intelligence and machine learning-based techniques are exclusively used in all domains, including clinical medicine [7,8]. Many soft computing techniques, namely expert systems, neural networks, fuzzy logic and genetic algorithms, have been established because they have advantages in solving ambiguity, imprecision and partial truth, even in the field of health $[9,10]$. FIS takes inputs and processes them to generate the outputs based on the preset values. The fuzzy controller's output is adopted using the identity membership function from fuzzifications of both inputs and outputs. A crisp input is translated into various identity membership functions based on its value. FIS performance is based on membership function; these membership functions can be evaluated as a collection of inputs [11,12]. More specifically, severe pneumonia leads to the coronavirus and coronavirus disease leads to death or kidney failure of a patient. Fuzzy set theory (FST), a powerful tool used to deal with imprecision and ambiguity, can tackle the idea of partially true and false values aimed at tractability, robustness, and low cost. The benefits of using fuzzy set theory carry accompanying characteristics and the ability to deal with linguistic terms that justify its use in solving medical problems and other application areas. FST offers a systematic calculation for linguistically handling knowledge and caries out numerical calculations using the language labels defined by membership functions. FST has become a significant mathematical approach to solve public health issues $[13,14]$.

FIS is the core unit of the fuzzy logic system, with decision making as its primary function. It uses the "IF... THEN" rules along with the "OR" or "AND" connectors to draw up basic guidelines for decision making [11]. Internet of Things (IoT) technology is becoming ever more 
common in the healthcare sector. IoT has proven its utility in the medical field with the advent of embedded devices, intelligent sensors and Web accessibility [15,16]. IoT's primary applications in intelligent medicine, including material management simulation, medical details, disease diagnosis, and medical process digitisation [17,18]. Sensors are used to monitor patient symptoms and help to manage a patient's health condition. IoT in every aspect of the healthcare industry is increasing day by day [19]. It is challenging to diagnose COVID-19 based on symptoms with similar diseases like flu, cough, fever, etc. In this study, we propose a system to diagnose the patients of COVID-19 based on symptoms. The system takes inputs as symptoms to derive disease. The system distinguished the different diseases according to the input symptoms. COVID-19 can be diagnosed through a Polymerase Chain Reaction test that analysed the virus based on a genetic fingerprint. Still, these types of tests are not feasible due to pandemic and quarantine restrictions. There is no specific medication for the COVID-19, and the treatment of the virus is compassionate care [3]. Generally, doctors and physicians diagnose the disease based on physical symptoms and laboratory test results. There is a dire need for a diagnosis system that can be applied efficiently and effectively so that an ordinary human being can do an early diagnosis and immediate treatment. The significant contributions of the paper are listed below:

- Proposes a smart diagnosis system to detect a disease that forms through multiple diseases.

- Helps to save time and money for expensive medicals tests.

- Helps in a life-critical situation by assisting medical practitioners in the early diagnosis of a disease.

This study aimed at developing an FIS using IoT for diagnosing the different kinds of diseases in the early stage.

\section{Related Work}

Fatima et al. [20] proposed an IoT based approach using FIS to intelligently and effectively predict COVID-19, known as IoT enabled coronavirus smart monitoring enabled by the fuzzy inference system (IoTSMCFIS), which smartly monitors and indicates whether or not human beings are victims of coronavirus. Avanzo et al. [21] suggested an extension of the system conceived for several inputs: Protein, red blood cells, lymphocytes, neutrophils, and eosinophils based on typical tumours, hemorrhage, and brain tumours. Since inputs are blood cells and the built device uses five blood cells for input, more than five inputs can be similarly defined to obtain an adequate diagnostic response. Future work into the fuzzy medical diagnostic control system is underway. Shehu et al. [22] developed the knowledge-based FIS for the diagnosis of Sepsis. The knowledge-based fuzzy system takes symptoms as inputs and predicts the output. Sepsis is a blood infection that causes damaged body tissues and organs. This system helped diagnose Sepsis in the early stages to prevent the bacteria from circulating in the whole body. Orozco-del-Castillo et al. [23] refined the FIS for analysis of Malaria and typhoid fever. Symptoms are taken as input, and output is the resulting disease. In this system, a person with or without risk of fever disease is also checked. This is the more comfortable and most effective approaches for diagnosing Malaria and typhoid fever.

Naseer et al. [24] proposed a decision support system based on a fuzzy logic system for heart disease detection, which predicts the possibilities of heart disease for the next 10 years. In this research work, risk factors that may cause heart disease are also analysed. The data contains 1230 instances of patients with normal and abnormal cases. For this prediction, fuzzy rules are designed. Different fuzzy clusters are created, and among these clusters, the best fuzzy decision rules are selected for the classification of patients. Umoh et al. [25] established a suggested 
Fuzzy Control and Cholera treatment system. The device configuration can be easily augmented, whichever a limit of inputs. Examples like membership functions like no cholera, mild cholera, moderate cholera, and severe cholera depend on inputs like diarrhea, vomiting, and dehydration. The system could be augmented to any number of inputs. To achieve efficiency in diagnosis and monitoring results, more inputs can be defined. Thakkar et al. [26] describe the prediction of diabetes disease with FIS so that users can easily use this application to perform the early action against diagnosis and rapid treatment. Al-Behadili et al. [27] define the uses of the Fuzzy decision process Diabetes based on Fuzzy logic. Diagnostic system for diagnosing diabetes. The fuzzy verdict mechanism applies the rules for determining whether or not a person has diabetes and summarises the results.

\section{Methodology}

The proposed system can be used to diagnose various diseases in a patient. The proposed system consists of two cloud computing aspects, as shown in Fig. 1. The first step defines the training phase, and the second step determines the validation phase. The training phase comprises three levels: the sensory layer, the data-preprocessing layer, and the final layer is the application layer. The Sensory layer comprises input parameters Fever, Cough, Headache, Respiratory Rate, Flu, Blood Pressure, Vomiting, and Diarrhea that assemble and bring the input values through IoT in the database. Because of the wireless communication of data, the data can be noisy and incomplete according to raw data type. After the sensory layer, the critical layer comes. Noise and Null values are handled by using the Normalisation and Moving Average technique. Data is transferred to the application layer after preprocessing. Application is categorised into two layers: Prediction and Performance layer.

FIS is used in the prediction layer to predict disease. Input is grouped and modified in a fuzzy set with fuzzy linguistics variables, fuzzy semantic points, and fuzzy membership functions. The FIS makes fuzzy modifications during the subsequent point. FIS is a way to presume given source input to use fuzzy logic after these fuzzy set possibilities are altered in a fuzzy set in a de-fuzzifier. This step is fundamental for a fuzzy control system. FIS is used to diagnose the disease in the prediction layer. If the inputs parameters in the sensory layers are relevant, they will move towards fuzzification into the fuzzy crisp inputs. The crisp set of input data is achieved and transformed into fuzzy sets using fuzzy linguistic variables and fuzzy membership functions. The fuzzy technologies continue over the FIS in the later step. FIS is the process to pretend input accustomed to the output using fuzzy logic, which is conclusively altered into crisp in defuzzy set consistency. The inputs parameter values are gathered to modify the fuzzy set with fuzzy grammatical variables, fuzzy semantic terms, and fuzzy membership functions. The fuzzy modifications make a FIS that predicts that the patient is healthy or not $[11,28,29]$.

\subsection{Membership Function}

Membership functions define the statistical explanation of input or output variables. The input variables are built for membership function values between the range of 0 and 1.

\subsection{Fuzzy Prepositions}

Here function t-norm is defined:

$t:[0,1] \times[0,1] \times[0,1] \times[0,1] \times[0,1] \times[0,1] \times[0,1] \times[0,1] \times[0,1] \rightarrow[0,1]$. 

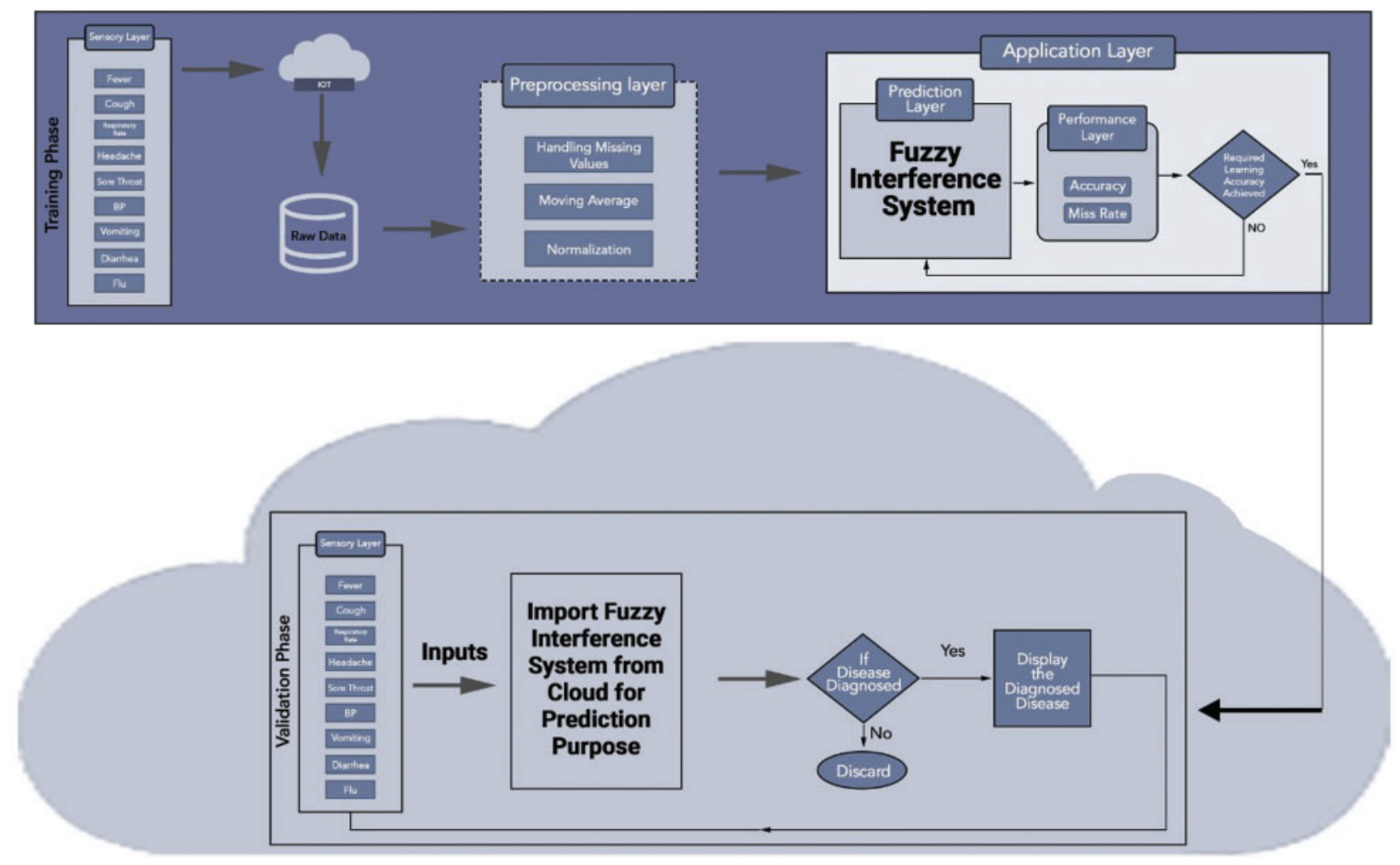

Figure 1: IoT enabled smart monitoring of diseases empowered with FIS

This equation alters the membership functions of fuzzy sets of Fever, Cough, Respiratory Rate, Headache, Sore Throat, Flu, Blood Pressure, and Diarrhea. We have defined some common methods which are used within the context of disease diagnosis. We have two phases for the disease diagnosis first phase is the training phase, and the second phase is the validation phase. We formulated the training phase as taking different diseases as input and sending it to the IoT layer. The raw data has been sent to the processing layer, where the outliers are removed from the data. Missing values are filled with mean, mod and average values. After the processing layer, the preprocessed data has been sent to the application layer; this layer comprises the prediction and performance layers. First, the cleaned data has been passed through the prediction layer. After the prediction layer, the performance layer evaluated the accuracy of the prediction. If the prediction rate is satisfied, then it will further be sent to the validation phase. In the validation phase, the system diagnoses disease from the information available in the cloud. Input data is sent to the Cloud FIS layer, and the disease has been predicted [30].

$$
\begin{aligned}
& t\left[\mu_{F}(f), \mu_{C}(c), \mu_{R R}(r r), \mu_{H}(h), \mu_{S T}(s t), \mu_{F}(f), \mu_{V}(v), \mu_{B P}(b p), \mu_{D}(d)\right] \\
& \quad=\min \left[\mu_{F}(f), \mu_{C}(c), \mu_{R R}(r r), \mu_{H}(h), \mu_{S T}(s t), \mu_{F}(f), \mu_{V}(v), \mu_{B P}(b p), \mu_{D}(d)\right]
\end{aligned}
$$

In Eq. (1) for the function t; qualifies for the intersection. For the intersection, the following must be satisfied and called t-norm. We also defined our proportion for t-norm. Either a person can have a disease or not, so based on this, we have discrete values 0,1 . In Eq. (2), we are 
qualified for intersection because Eq. (1) satisfying the Axiom tl can quickly implement the min function of fuzzy.

\section{Axiom t1: (Boundary Condition)}

$t(0,0)=0$ or $\Lambda[0,0]=0$;

$\mathrm{T}\left[\mu_{A}(x), 1\right]=T\left[1,\left[\mu_{A}(x)\right]=\mu_{A}(x)\right.$

Axiom t2: (Commutativity)

$\mathrm{T}\left[\mu_{A}(x), \mu_{B}(x)\right]=T\left[\mu_{B}(x),\left[\mu_{A}(x)\right]\right.$

Axiom t3: (Non-Decreasing)

If $\mu_{A}(x) \leqslant \mu_{B}(x)$ and $\mu_{C}(x) \leqslant \mu_{D}(x)$ then $\mathrm{T}\left[\mu_{A}(x), \mu_{C}(x)\right] \leqslant\left[\mu_{B}(x), \mu_{D}(x)\right]$

Axiom t4: (Associativity)

$\mathrm{T}\left[\mathrm{T}\left[\mu_{A}(x), \mu_{B}(x)\right], \mu_{C}(x)\right]\left[\mu_{A}(x), T\left[\mu_{B}(x), \mu_{C}(x)\right]\right]$

Eq. (3) explained the membership function as following:

$\mu_{F \cap C \cap R R \cap H \cap S T \cap F \cap V \cap B P \cap D}(f, c, r r, h, s t, f, v, b p, d)$

$=t\left[\mu_{F}(f), \mu_{C}(c), \mu_{R R}(r r), \mu_{H}(h), \mu_{S T}(s t), \mu_{F}(f), \mu_{V}(v), \mu_{B P}(b p), \mu_{D}(d)\right]$

Eq. (4) elaborated the fuzzy intersection function.

$\mu_{F \cap C \cap R R \cap H \cap S T \cap F \cap V \cap B P \cap D}(f, c, r r, h, s t, f, v, b p, d)$

$=\min \left[\mu_{F}(f), \mu_{C}(c), \mu_{R R}(r r), \mu_{H}(h), \mu_{S T}(s t), \mu_{F}(f), \mu_{V}(v), \mu_{B P}(b p), \mu_{D}(d)\right]$

Eq. (5) explains the fuzzy IF-THEN rule that is inferred as a fuzzy relation with membership function:

$$
\begin{aligned}
& \mu Q(f, c, r r, h, s t, f, v, b p, d) \\
& \quad=\min \left[\mu_{F P 1}(f), \mu_{F P 2}(c), \mu_{F P 3}(r r), \mu_{F P 4}(h), \mu_{F P 5}(s t), \mu_{F P 6}(f), \mu_{F P 7}(v), \mu_{F P 8}(b p), \mu_{F P 9}\right]
\end{aligned}
$$

\subsection{Fuzzy Inference Engine}

The logic concepts are inured in fuzzy inference to associate fuzzy IF-THEN rules in fuzzy rule base into outlining from a fuzzy input set to a fuzzy output set. In input-output product space, the IF-THEN rule is interpreted as a fluid relationship that defines the fuzzy relationship. Any practical base of rules is more than one; the main problem here is how to deduce a collection of rules. There are two ways of inferring rule collection: inference based on composition and inference based on the individual rule. Through composition-based inference, all rules in fuzzy rule base merged in a single fuzzy relationship which rests on input universes of discourse under the inner product and is then treated as a single fuzzy IF-THEN rule [31].

\subsection{De-Fuzzifier}

De-Fuzzification is an approach for making a computable conclusion in plump logic, accepted fuzzy sets and comparing membership degrees. The technique makes a fuzzy set a fresh set that is also required in fuzzy control frameworks. There are various kinds of De-Fuzzifier. In the proposed model, a centroid kind of De-Fuzzifier is applied [32]. The figures describe the De-Fuzzifier graphical description. Figs. 2-4 show the De-Fuzzifier graphic description of FIS. 


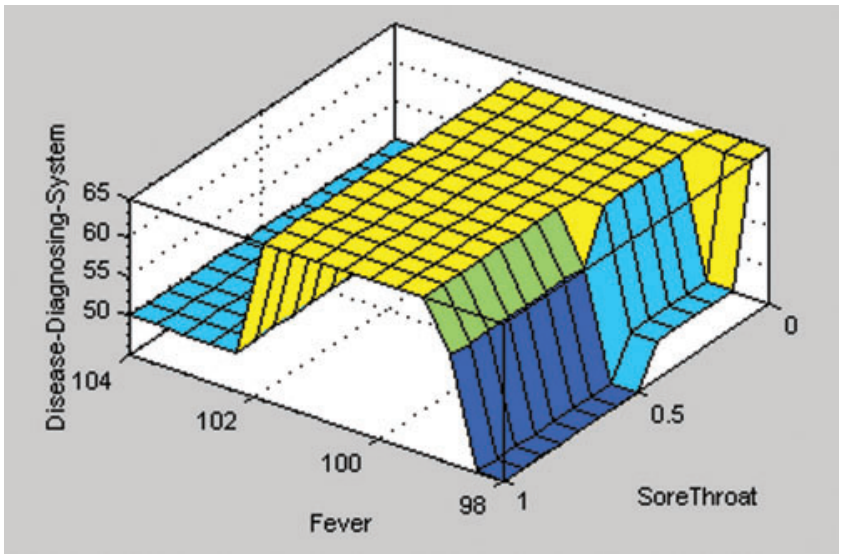

Figure 2: Rule surface of the proposed system based on sore throat and fever

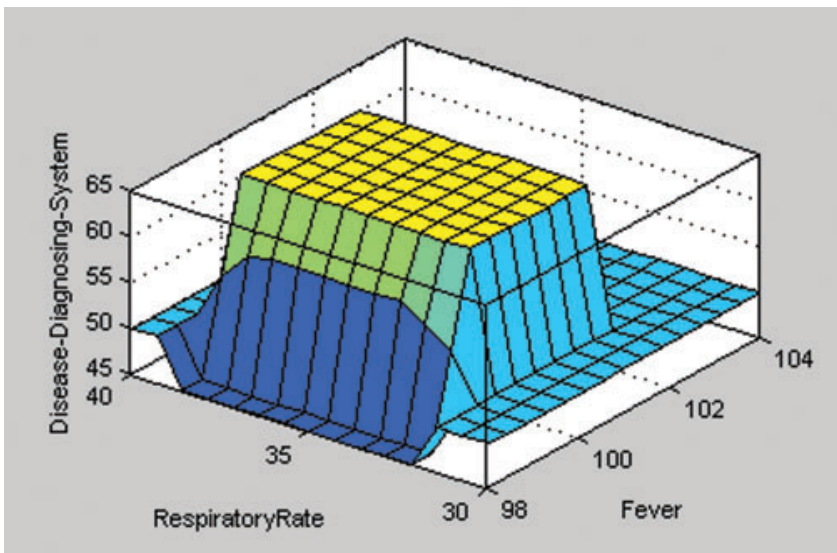

Figure 3: Rule surface of the proposed system based on fever and respiratory rate

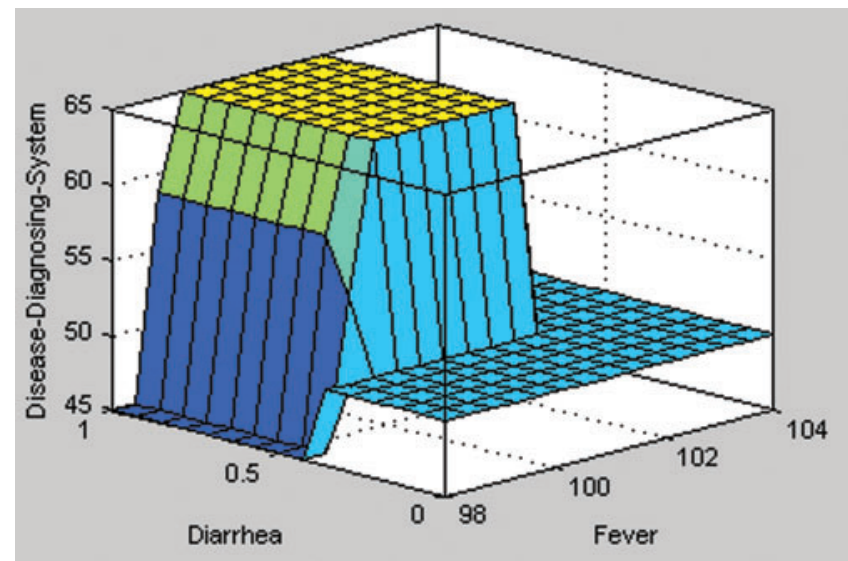

Figure 4: Rule surface of the proposed system based on fever and diarrhea 


\section{Results}

The fuzzy Knowledge base is built in MATLAB using the Fuzzy Logic Toolkit. The toolkit is an application suite that forms a MATLAB environment. This toolkit enables to design of fuzzy inference structure and fuzzy classification in MATLAB context, i.e., performance guided to shape flexible allocation for data systems. The base component in Compilation is FIS-structure, i.e., the FIS. The FIS consists of useful blocks necessary to implement the fuzzy inference. System of medical diagnosis take inputs in the form of symptoms and produce output in the shape of a given illness. Fuzzy sets are a distinguishing method for the various kinds of information linked with the information and indicate the connections and sources between their variables. The proposed model produces Input and Output rules membership functions present below. Below appeared the FIS suggests rules.

(1) IF (Fever is LOW), (Cough is Low), (Respiratory Rate is Low), (Headache is Moderate) and (Sore Throat is Low), (FLU is No), (Vomiting is No), (Blood Pressure is Low), and (Diarrhea is No) THEN (Disease-Diagnosing-System is Malaria)

(2) IF (Fever is LOW), (Cough is Low), (Respiratory Rate is Low), (Headache is Moderate), (Sore Throat is Low), (FLU is No) and (Vomiting is No), (Blood Pressure is Low), and (Diarrhea is No) THEN (Disease-Diagnosing-System is Malaria)

(3) IF (Fever is LOW), (Cough is Low), (Respiratory Rate is Low), (Headache is Moderate), (Sore Throat is Low), (FLU is No), (Vomiting is No) and (Blood Pressure is Low), and (Diarrhea is No) THEN (Disease-Diagnosing-System is Malaria)

(4) IF (Fever is LOW), (Cough is Low), (Respiratory Rate is Low), (Headache is Moderate), (Sore Throat is Low), (FLU is No), (Vomiting is No), (Blood Pressure is Low), and (Diarrhea is No) THEN (Disease-Diagnosing-System is Malaria)

(5) IF (Fever is High), (Cough is High), (Respiratory Rate is High), (Headache is Severe), (Sore Throat is High), (FLU is Yes), (Vomiting is Yes), (Blood Pressure is High), and (Diarrhea is Yes) THEN (Disease-Diagnosing-System is Coronavirus)

Fig. 2 shows a 3D view of the proposed system's ruled surface depends on the Sore Throat and Fever. It is noted the findings of the presented system results are good with yellow shade. If fever is from 99 and 102 and sore throat lies between 0 to 0.25 . If the fever lies between 98.5 to 99.5 and the sore throat lies between 0.5 to 1 , it shows average results. If fever lies between 98.5 to 98 and sore throat lies between 0.5 to 1 , it shows terrible results with a bluish shade.

Fig. 3 shows the 3D view of the proposed system's ruled surface depends on the Respiratory Rate and Fever. If the fever range is between 100.5 to 102 and the respiratory rate lies between 31 to 35 , it shows Good results. If the fever lies between 100 to 100.5 and respiratory rate between 31 to 33 , it shows average results.

Fig. 4 shows the 3D view of the proposed system ruled surface depends on the Fever and Diarrhea. It is noted that the finding of the proposed system is promising if fever lies between 101 and 104 and diarrhea from 0.5 to 1 . Suppose will show average results if fever lies between 100 and 101 and diarrhea between 0.4 to 0.6. It will show bad results if fever lies between 98 to 100 and diarrhea between 0.5 to 1 . After the prediction layer, the output will be sent to the performance layer in which the value is checked in terms of accuracy and miss rate and also it is checked that if the performance is not according to the learning criteria, then the prediction layer will be updated and in case of Yes, the value is stored on the cloud database. Then it further moves to the validation phase to import Fuzzy based smart disease diagnosing system, and it is checked if the disease is diagnosed. The system will display the message of a diagnosed disease. 
In the case of no, the process will be discarded. The input/output variables of the membership function with the fuzzy system are stated in Tab. 1.

Table 1: Input/output variables of membership function with fuzzy system: $\mathrm{X}$-axis represents the input variables like cough, fever, respiratory rate, and headache; The y-axis represents the degrees of membership in the $[0,1]$ interval

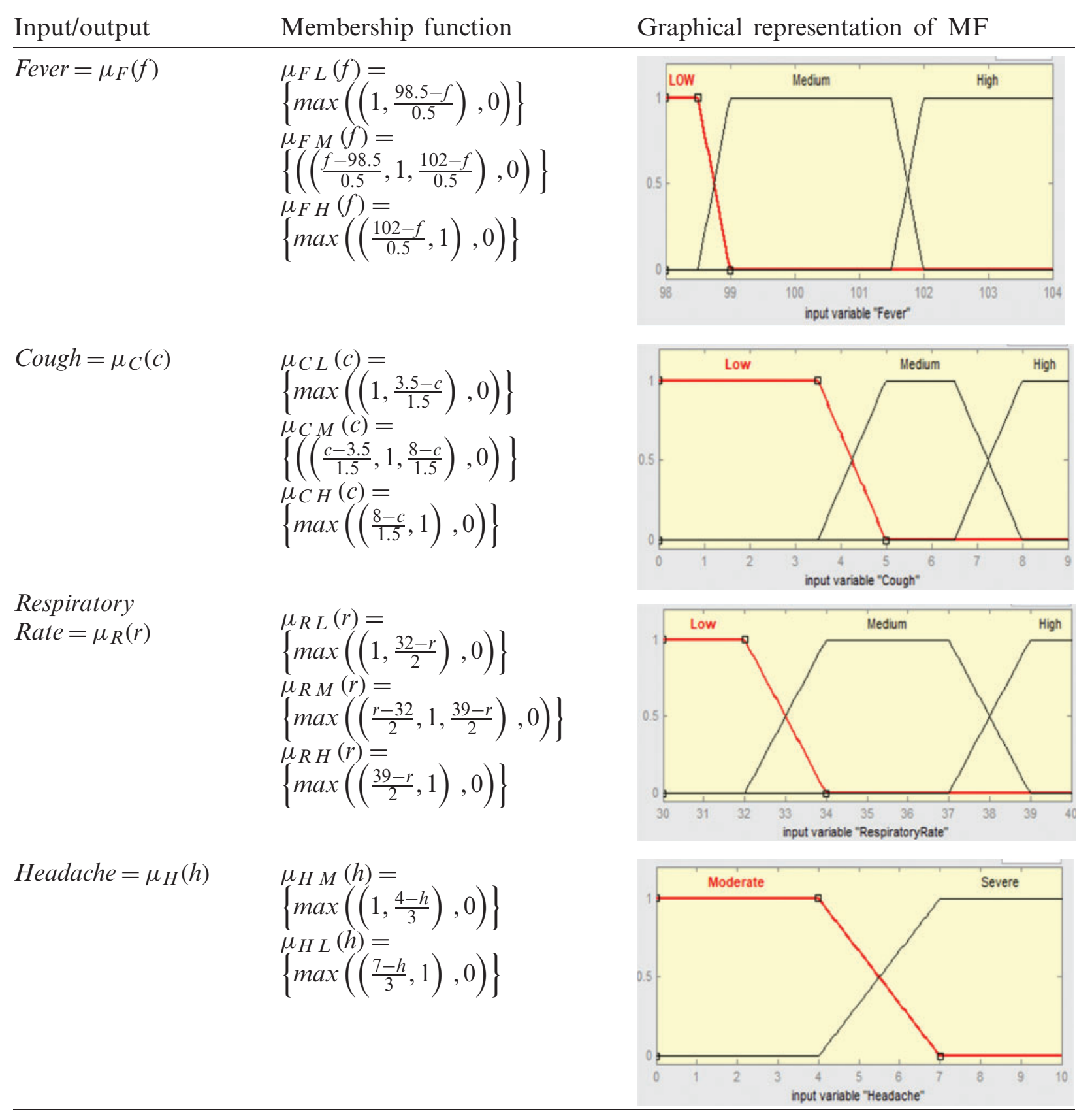

(Continued) 
Table 1: Continued

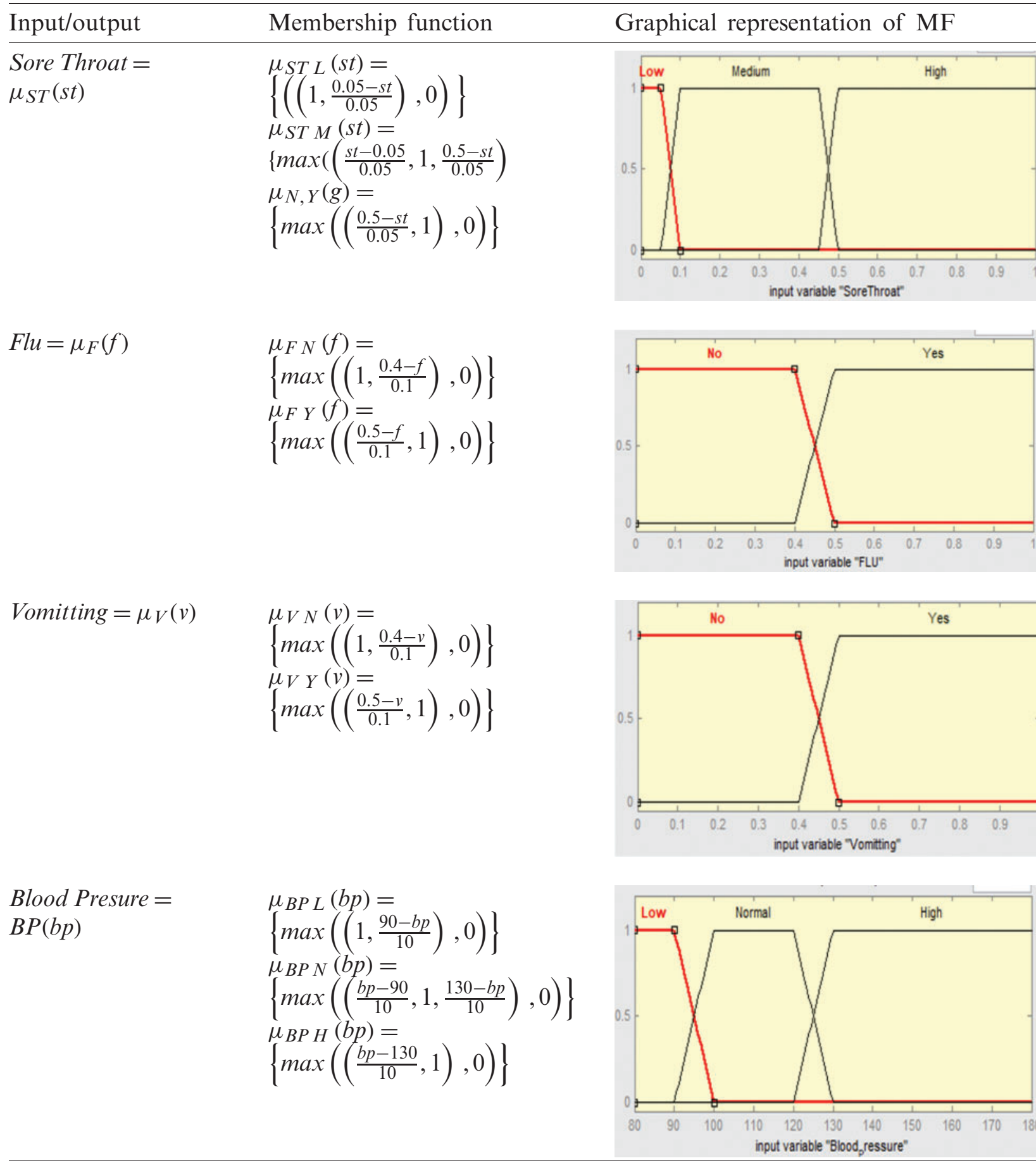

(Continued) 
Table 1: Continued

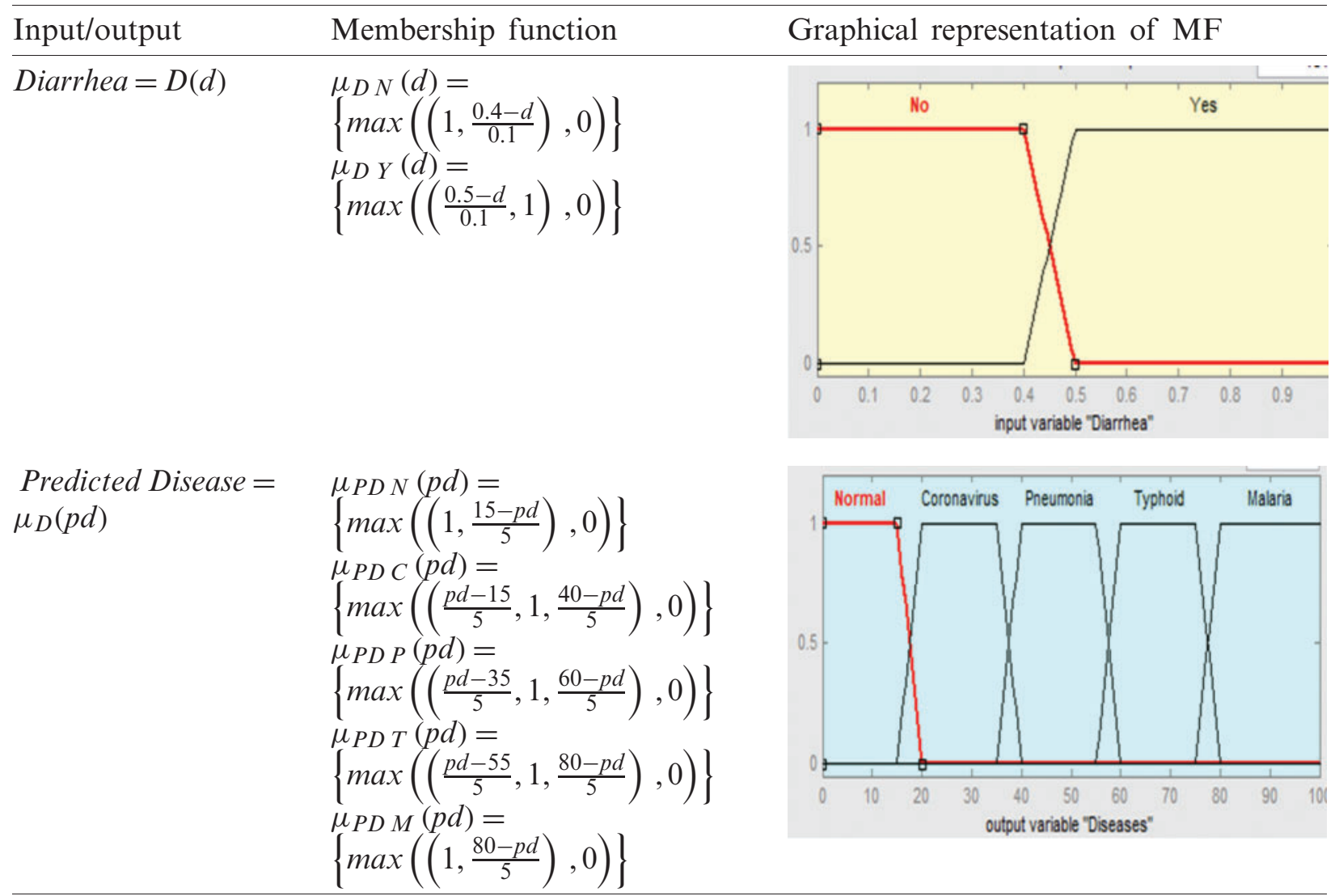

MATLAB is applied for simulation with nine inputs and one output parameter. The proposed system establishes various types of output. Figs. 5-9, respectively, shows the lookup rules diagram of the proposed system. Fig. 5 shows that if fever values are low, headache is moderate, respiratory rate is low, cough is low, headache is moderate, sore throat is low, flu is no, blood pressure is low, vomiting is no. Diarrhea is no, and then the patient is healthy.

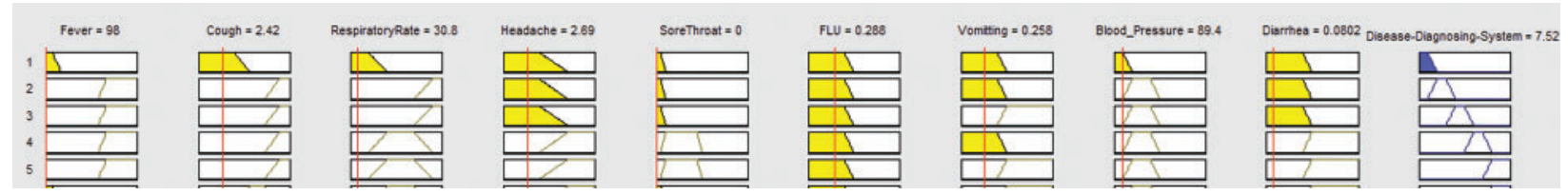

Figure 5: Lookup rule diagram for proposed system (Healthy)

Fig. 6 displays if fever values are High, Headache is Moderate, Respiratory Rate is High, Cough is High, Headache is Moderate, Sore throat is Low, Flu is No, Blood Pressure is Low, Vomiting is No. Diarrhea is No, and then the patient has pneumonia. 


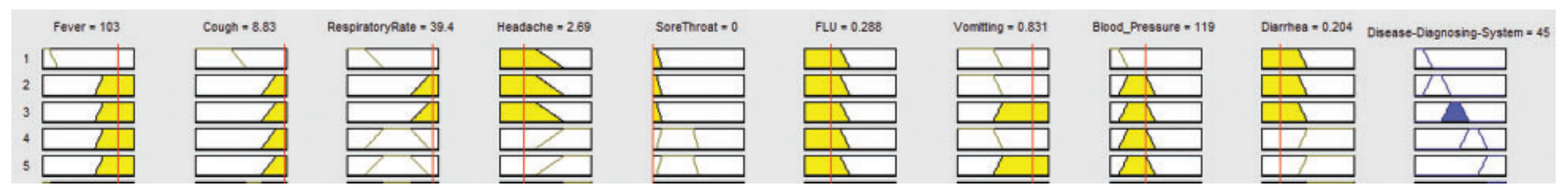

Figure 6: Lookup rule diagram for the proposed system (Pneumonia)

Fig. 7 displays if values of Fever are Low, Headache is Low, Respiratory Rate is Normal, Cough is High, Headache is Moderate, Sore throat is Low, Flu is No, Blood Pressure is Normal, Vomiting is Yes, and Diarrhea is Yes then Patient having Malaria.

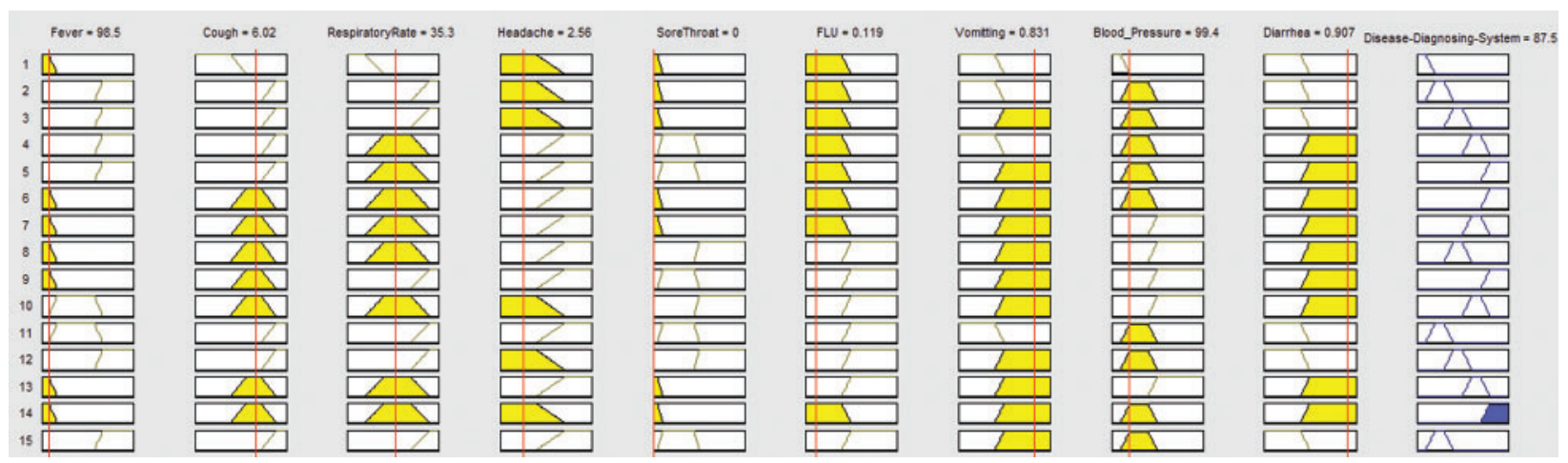

Figure 7: Lookup rule diagram for the proposed system (Malaria)

Fig. 8 displays if values of Fever are High, Headache is Low, Respiratory Rate is High, Cough is High, Headache is Moderate, Sore throat is Low, Flu is No, Blood Pressure is Normal, Vomiting is No. Diarrhea is No, then the patient having coronavirus.

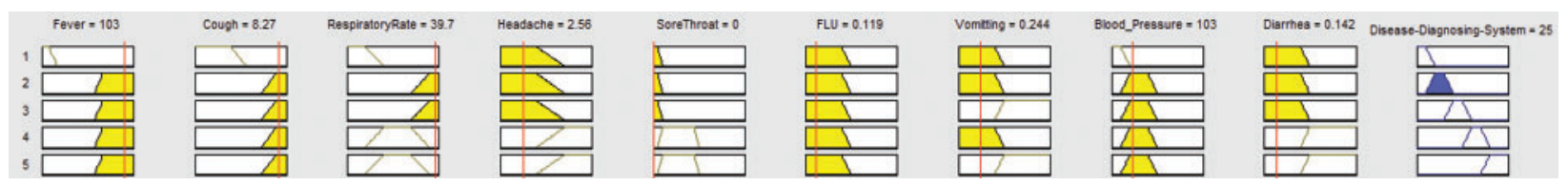

Figure 8: Lookup rule diagram for the proposed system (Coronavirus)

Fig. 9 displays if values of Fever are Low, Headache is Low, Respiratory Rate is High, Cough is High, Headache is Moderate, Sore throat is Low, Flu is No, Blood Pressure is Normal, Vomiting is No. Diarrhea is No, then the patient having Typhoid. 


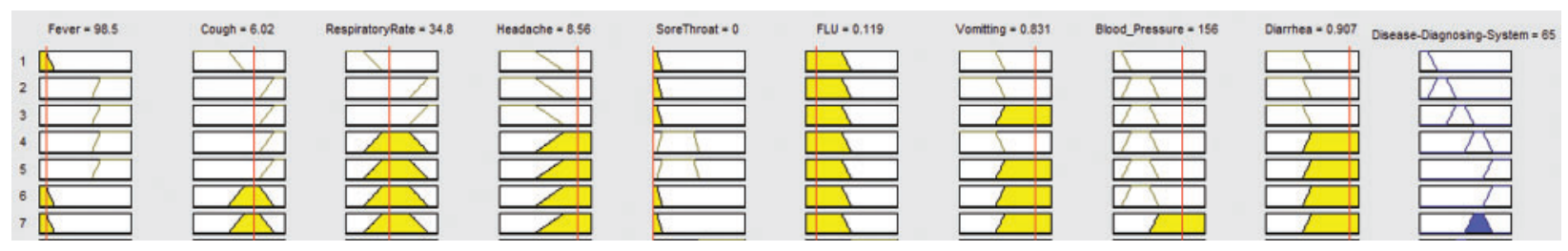

Figure 9: Lookup rule diagram for the proposed system (Typhoid)

\section{Conclusion}

The research aimed to build a system that could help experts or medical practitioners in preliminary diagnosing. Fuzzy set theory plays a crucial role in dealing with ambiguity while deciding on medical applications. The use of fuzzy logic allows us to use uncertainty in the classifier design and consequently increase the integrity of the developed system output. The Fuzzy classifier was used to predict the multiple diseases when ten features of various diseases were taken as input. The illustrated model combined the FIS and IoT. The classified results and statistical measures were used for the evaluation of this model. The obtained results of the proposed model can be used in the prediction of multiple diseases by taking into consideration the misclassification rates. Acquiring the data is the biggest challenge to perform this research. In the future, it is desirable to obtain a large dataset and apply the same methodology and compare the new results to the results obtained in this study. The goal is to develop a FIS that can be used in the real world. The future work of this research work extends the approach towards a diagnosis of severe diseases other than COVID-19, such as diabetes, liver diseases, kidney diseases, etc. Furthermore, Fuzzy controllers may develop expert systems in different areas such as bioinformatics, traffic signals, biological diagnosis, power system control, etc.

Funding Statement: The authors received no specific funding for this study.

Conflicts of Interest: The authors declare that they have no conflicts of interest to report regarding the present study.

\section{References}

[1] H. Lu, C. W. Stratton and Y. W. Tang "Outbreak of pneumonia of unknown etiology in Wuhan, China: The mystery and the miracle," Journal of Medical Virology, vol. 92, pp. 401-402, 2020.

[2] D. Wang, B. Hu, C. Hu, F. Zhu, X. Liu et al., "Clinical characteristics of 138 hospitalised patients with 2019 novel coronavirus-infected pneumonia in Wuhan, China," JAMA, vol. 323, pp. 1061-1069, 2020.

[3] A. Nasir, K. Shaukat, I. A. Hameed, S. Luo, T. Mahboob et al., "A bibliometric analysis of corona pandemic in social sciences: A review of influential aspects and conceptual structure," IEEE Access, vol. 8, pp. 133377-133402, 2020.

[4] D. S. Hui and A. Zumla, "Severe acute respiratory syndrome: Historical, epidemiologic, and clinical features," Infectious Disease Clinics, vol. 33, pp. 869-889, 2019.

[5] S. Platto, J. Zhou, Y. Wang, H. Wang and E. Carafoli "Biodiversity loss and COVID-19 pandemic: The role of bats in the origin and the spreading of the disease," Biochemical and Biophysical Research Communications, vol. 538, pp. 2-13, 2021.

[6] A. H. Khan, M. Hussain and M. K. Malik "ECG images dataset of cardiac and COVID-19 patients," Data in Brief, vol. 34, pp. 106762, 2021. 
[7] K. Shaukat, T. M. Alam, M. Ahmed, S. Luo, I. A. Hameed et al., "A model to enhance governance issues through opinion extraction," in 2020 11th IEEE Annual Information Technology, Electronics and Mobile Communication Conf. (IEMCON), Vancouver, BC, Canada, pp. 511-516, 2020.

[8] T. M. Alam, K. Shaukat, H. Mahboob, M. U. Sarwar, F. Iqbal et al., "A machine learning approach for identification of malignant mesothelioma etiological factors in an imbalanced dataset," The Computer Journal, vol. 64, pp. 82424, 2020.

[9] S. Kamran, I. Farhat, A. Talha Mahboob, A. Gagandeep Kaur, D. Liton et al., "The impact of artificial intelligence and robotics on the future employment opportunities," Trends in Computer Science and Information Technology, vol. 5, pp. 5, 2020.

[10] K. Shaukat, T. M. Alam, I. A. Hameed, S. Luo, J. Li et al., "A comprehensive dataset for bibliometric analysis of SARS and coronavirus impact on social sciences," Data in Brief, vol. 33, pp. 106520, 2020.

[11] D. Karaboga and E. Kaya, "Adaptive network based fuzzy inference system (ANFIS) training approaches: A comprehensive survey," Artificial Intelligence Review, vol. 52, pp. 2263-2293, 2019.

[12] A. H. Khan, M. Hussain and M. K. Malik, "Cardiac disorder classification by electrocardiogram sensing using deep neural network," Complexity, vol. 2021, pp. 5512243, 2021.

[13] K. Mokhtari, J. Ren, C. Roberts and J. Wang, "Decision support framework for risk management on sea ports and terminals using fuzzy set theory and evidential reasoning approach," Expert Systems with Applications, vol. 39, pp. 5087-5103, 2012.

[14] J. Xu, L. Guo, R. Zhang, H. Hu, F. Wang et al., "QoS-aware service composition using fuzzy set theory and genetic algorithm," Wireless Personal Communications, vol. 102, pp. 1009-1028, 2018.

[15] K. T. Kadhim, A. M. Alsahlany, S. M. Wadi and H. T. Kadhum, "An overview of patient's health status monitoring system based on internet of things (IoT)," Wireless Personal Communications, vol. 114, pp. 2235-2262, 2020.

[16] A. H. Khan, M. Hussain and M. K. Malik, "Arrhythmia classification techniques using deep neural network," Complexity, vol. 2021, pp. 9919588, 2021.

[17] K. Shaukat, S. Luo, N. Abbas, T. M. Alam, M. E. Tahir et al., "An analysis of blessed Friday sale at a retail store using classification models," in Presented at the 2021 the 4th Int. Conf. on Software Engineering and Information Management, Yokohama, Japan, 2021.

[18] T. M. Alam, K. Shaukat, I. A. Hameed, W. A. Khan, M. U. Sarwar et al., "A novel framework for prognostic factors identification of malignant mesothelioma through association rule mining," Biomedical Signal Processing and Control, vol. 68, pp. 102726, 2021.

[19] Y. Zhang, G. Chen, H. Du, X. Yuan, M. Kadoch et al., "Real-time remote health monitoring system driven by 5G MEC-IoT," Electronics, vol. 9, pp. 1753, 2020.

[20] S. A. Fatima, N. Hussain, A. Balouch, I. Rustam, M. Saleem et al., "IoT enabled smart monitoring of coronavirus empowered with fuzzy inference system," International Journal of Advance Research, Ideas and Innovations in Technology, vol. 6, pp. 188-194, 2020.

[21] M. Avanzo, L. Wei, J. Stancanello, M. Vallieres, A. Rao O. Morin et al., "Machine and deep learning methods for radiomics," Medical Physics, vol. 47, pp. e185-e202, 2020.

[22] N. Shehu, J. Bimba, D. Nantok, I. Kelly, O. Obrerhor et al., "Over diagnosis of typhoid and malaria co-infection by health care workers in north central Nigeria: A cross-sectional study," Nigerian Journal of Medicine, vol. 29, pp. 100-103, 2020.

[23] M. Orozco-del-Castillo, R. Novelo-Cruz, J. Hernández-Gómez, P. Mena-Zapata, E. Brito-Borges et al., "Fuzzy logic-based covid-19 and other respiratory conditions pre-clinical diagnosis system," in Int. Congress of Telematics and Computing, Mexico, pp. 402-419, 2020.

[24] I. Naseer, B. S. Khan, S. Saqib, S. N. Tahir, S. Tariq et al., "Diagnosis heart disease using mamdani fuzzy inference expert system," EAI Endorsed Transactions on Scalable Information Systems, vol. 7, pp. $1-9,2020$.

[25] U. A. Umoh and M. M. Ntekop, "A proposed fuzzy framework for cholera diagnosis and monitoring," International Journal of Computer Applications, vol. 82, pp. 1-10, 2013. 
[26] H. Thakkar, V. Shah, H. Yagnik and M. Shah "Comparative anatomisation of data mining and fuzzy logic techniques used in diabetes prognosis," Clinical EHealth, vol. 4, pp. 12-23, 2020.

[27] H. N. K. Al-Behadili and K. R. Ku-Mahamud, "Fuzzy unordered rule using greedy hill climbing feature selection method: An application to diabetes classification," Journal of Information and Communication Technology, vol. 20, pp. 391-422, 2021.

[28] N. Chandgude and S. Pawar, "Diagnosis of diabetes using fuzzy inference system," in 2016 Int. Conf. on Computing Communication Control and Automation (ICCUBEA), India, pp. 1-6, 2016.

[29] W. M. Shaban, A. H. Rabie, A. I. Saleh and M. Abo-Elsoud, "Detecting COVID-19 patients based on fuzzy inference engine and deep neural network," Applied Soft Computing, vol. 99, pp. 106906, 2021.

[30] H. Garg and D. Rani, "New generalised Bonferroni mean aggregation operators of complex intuitionistic fuzzy information based on archimedean t-norm and t-conorm," Journal of Experimental \& Theoretical Artificial Intelligence, vol. 32, pp. 81-109, 2020.

[31] M. W. Nadeem, M. Hussain, M. A. Khan and S. M. Awan, "Analysis of smart citizens: A fuzzy based approach," in 2019 Int. Conf. on Electrical, Communication, and Computer Engineering (ICECCE), Malaysia, pp. 1-5, 2019.

[32] M. W. Nadeem, M. Hussain, M. A. Khan, M. U. Munir and S. Mehrban, "Fuzzy-based model to evaluate city centric parameters for smart city," in 2019 Int. Conf. on Innovative Computing (ICIC), Pakistan, pp. 1-7, 2019. 